\title{
An improved scheme for modeling the reflectance spectra of space-weathered regoliths
}

\author{
Tokuhiro Nimura ${ }^{1,2,3}$, Takahiro Hiroi ${ }^{1}$, and Carle M. Pieters ${ }^{1}$ \\ ${ }^{1}$ Department of Geological Sciences, Brown University, Providence, RI 02912, USA \\ ${ }^{2}$ Department of Earth and Planetary Science, The University of Tokyo, 7-3-1 Hongo, Bunkyo-ku, Tokyo 113-0033, Japan \\ ${ }^{3}$ Department of Planetary Science, Institute of Space and Astronautical Science, Japan Aerospace Exploration Agency, \\ 3-1-1 Yoshinodai, Sagamihara, Kanagawa 229-8510, Japan
}

(Received March 31, 2007; Revised November 6, 2007; Accepted November 25, 2007; Online published April 9, 2008)

In the remote detection of mineral compositions of planetary surface materials on airless bodies, space weathering is an obstacle which makes reflectance spectra redder and darker and weakens spectral features. This study attempts to model an effect of space weathering due to vapor coating by accounting for the change in surface reflectivity of regolith particles wherein nanophase-reduced iron $\left(\mathrm{npFe}^{0}\right)$ particles are concentrated on their surfaces. When applied to a suite of reflectance spectra of lunar soils having different degrees of space weathering, this model can estimate the degrees of space weathering in terms of the thicknesses of the coating layer and provide an absorption coefficient spectrum of the host material.

Key words: Space weathering, airless bodies, vapor coating, surface reflectivity, regolith particles, modeling.

\section{Introduction}

Visible and near-infrared reflectance spectroscopy has been a useful method for remotely detecting mineralogy of planetary surface materials. However, in the case of airless bodies, space weathering effects may be so strong that their surface reflectance spectra show reddened continua, lowered albedo, and attenuated absorption features (e.g., Pieters et al., 1993), all of which make it more difficult to use important diagnostic features for detecting component minerals and their chemical compositions. Space weathering is a process that alters the state of surface materials exposed to the harsh space environment. Surfaces of airless bodies are subject to the irradiation of solar and galactic cosmic rays and solar wind, which cause ion implantation and sputtering, and to bombardment by all sizes of meteorites and micrometeorites. As a result, the exposed surfaces develop radiation damage, vapor- and sputter-deposited coatings, and melt products, including agglutinates.

One form of space weathering products is a vapor coating containing nanophase-reduced iron $\left(\mathrm{npFe}^{0}\right)$ particles around each regolith particle. Transmission electron microscope (TEM) images have revealed the details of $n p \mathrm{Fe}^{0}$ layering along the rims of lunar soil grains (e.g., Keller and McKay, 1993, 1997; Wentworth et al., 1999). The simulation of micrometeorite bombardment has been proven to form a vapor-deposited layer containing $\mathrm{npFe}^{0}$ particles (Sasaki et al., 2001). Hapke (2001) modeled the optical effect of $n p \mathrm{Fe}^{0}$ particles in a semi-transparent matrix. The purpose of this study is to create an improved model for this space weathering process that is able to account for its

Copyright (c) The Society of Geomagnetism and Earth, Planetary and Space Sciences (SGEPSS); The Seismological Society of Japan; The Volcanological Society of Japan; The Geodetic Society of Japan; The Japanese Society for Planetary Sciences; TERRAPUB. effect on the boundary reflectivity of regolith particles and to subsequently apply this model to reflectance spectra of lunar soils.

\section{Modeling}

The basic scheme of our new model is depicted in Fig. 1. Each regolith particle consists of a host mineral of $d_{h}$ in diameter, covered with a vapor coating layer of $d_{w}$ in thickness, containing $n \mathrm{pFe}^{0}$ particles by a volume fraction of $\phi_{w}$. They are formed in an amorphous matrix having an assumed refractive index (the real part $n_{h}$ only). Real and imaginary refractive indices, $n_{w}$ and $k_{w}$, respectively, of that layer are calculated based on Hapke's space weathering model, which employs the Maxwell-Garnett effective medium model (Hapke, 2001):

$$
\begin{gathered}
K_{w}=K_{h}\left(1+\frac{3 \phi_{w} \frac{K_{\mathrm{Fe}}-K_{h}}{K_{\mathrm{Fe}}+2 K_{h}}}{1-\phi_{w} \frac{K_{\mathrm{Fe}}-K_{h}}{K_{\mathrm{Fe}}+2 K_{h}}}\right), \\
K_{w}=\left(n_{w}+i k_{w}\right)^{2}, K_{h}=\left(n_{h}+i k_{h}\right)^{2}, K_{\mathrm{Fe}}=\left(n_{\mathrm{Fe}}+i k_{\mathrm{Fe}}\right)^{2},
\end{gathered}
$$

where $n$ and $k$ indicate real and imaginary parts of the refractive index respectively, and they are indicated with appropriate suffixes, wherein $w, h$, and Fe indicate vapor coating, host material, and Fe, respectively. Then the absorption coefficient of the vapor coating layer is given by:

$$
\alpha_{w}=4 \pi k_{w} / \lambda \text {. }
$$

Figure 2 depicts a scheme to model the reflection and transmission of light at the boundaries of the vapor coating layer on a regolith particle. Because the phases of boundary reflectivity $(r)$ of the inner and outer boundaries of the coating layer in two different directions are different from each 


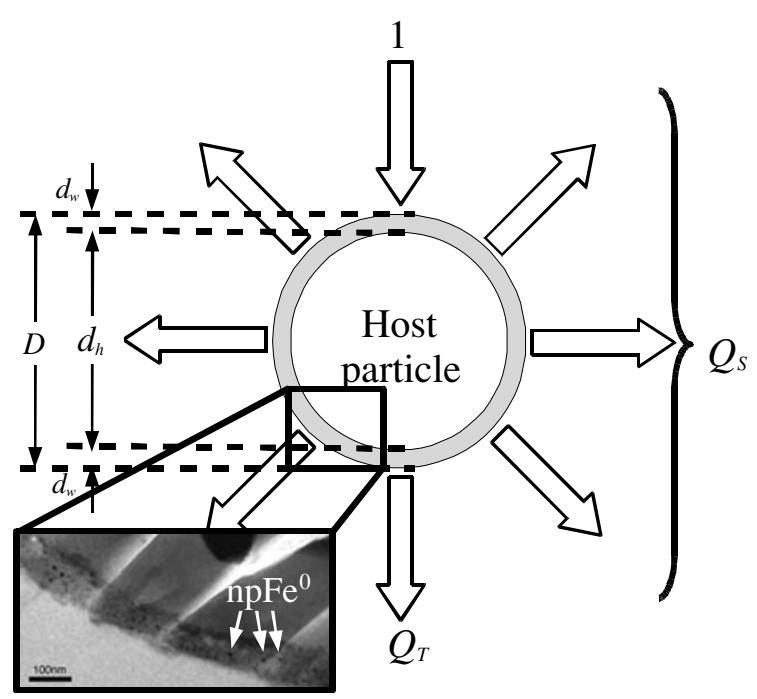

Fig. 1. A schematic view of a space-weathered particle. $D, d_{h}$, and $d_{w}$ denote the diameters of the whole particle, the host particle, and the weathering layer, respectively. Inset: Transmission electron microscopy bright-field image of a lunar plagioclase grain with a $\mathrm{nFe}^{0}$-rich coating (Noble et al., 2002). $Q_{S}$ and $Q_{T}$ denote scattering and transmitting efficiencies, respectively.

other, these are indicated with the appropriate suffixes, with $0, w$, and $h$ indicating the vacuum, vapor coating layer, and host mineral, respectively. These boundary reflectivities are calculated from the refractive indices of these three phases using Fresnel Theory and integrating them over all incident angles. Total scattering $(s)$ and transmitting $(t)$ activities of this layer can be calculated using these boundary reflectivities as well as the absorption coefficient $\left(\alpha_{w}\right)$ and thickness $\left(d_{w}\right)$ of the vapor coating layer:

$$
\begin{aligned}
s_{0 w} & =r_{0 w}+\frac{\left(1-r_{0 w}\right)\left(1-r_{w 0}\right) r_{w h} p_{w}^{2}}{1-r_{w 0} r_{w h} p_{w}^{2}}, \\
t_{0 w} & =\frac{\left(1-r_{0 w}\right)\left(1-r_{w h}\right) p_{w}}{1-r_{w 0} r_{w h} p_{w}^{2}}, \\
s_{h w} & =r_{h w}+\frac{\left(1-r_{h w}\right)\left(1-r_{w h}\right) r_{w 0} p_{w}^{2}}{1-r_{w 0} r_{w h} p_{w}^{2}}, \\
t_{h w} & =\frac{\left(1-r_{h w}\right)\left(1-r_{w 0}\right) p_{w}}{1-r_{w 0} r_{w h} p_{w}^{2}}, \\
p_{w} & =\exp \left(-\alpha_{w} d_{w}\right) .
\end{aligned}
$$

Figure 3 shows a scheme for calculating the scattering and transmitting efficiencies of a space-weathered particle with host mineral absorption coefficient $\alpha_{h}$ and coating thickness $d_{h}$. According to the scheme of Hiroi and Pieters (1994), when Hapke's model (Hapke, 1981) is used, a part of the unit incident light onto a grain is transmitted without being reflected (transmitting efficiency $Q_{T}$ ), another part is scattered to other directions $\left(Q_{S}\right)$, and the rest is absorbed. Extinction efficiency $Q_{E}$ is defined as the portion of unit incident light attenuated by a single particle. Thus, $Q_{E}$ is formulated here by excluding the transmitting efficiency $\left(Q_{T}\right)$ as:

$$
Q_{E}=1-Q_{T}
$$

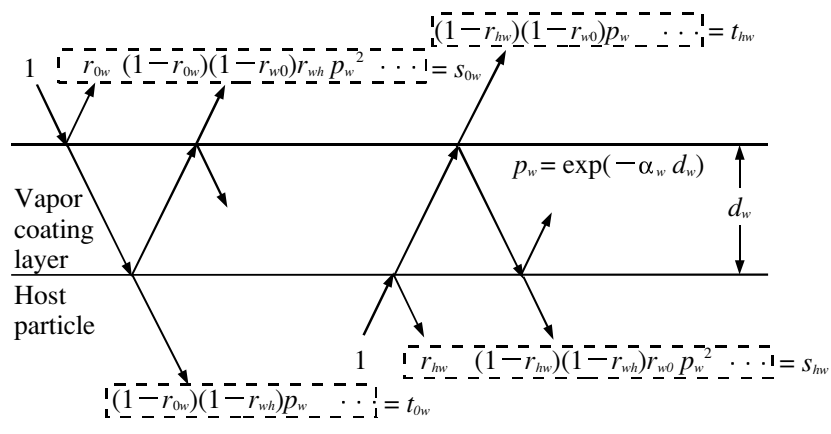

Fig. 2. A scheme for modeling the process of light scattering near a vapor-coating layer. External and internal reflectances $\left(s_{0 w}\right.$ and $\left.s_{h w}\right)$ and external and internal transmittances $\left(t_{0 w}\right.$ and $\left.t_{h w}\right)$ of the particle are calculated from boundary reflectivities $\left(r_{0 w}, r_{w 0}, r_{w h}\right.$, and $\left.r_{h w}\right)$, the absorption coefficient $\alpha_{w}$, and thickness $d_{w}$ of the coating layer.

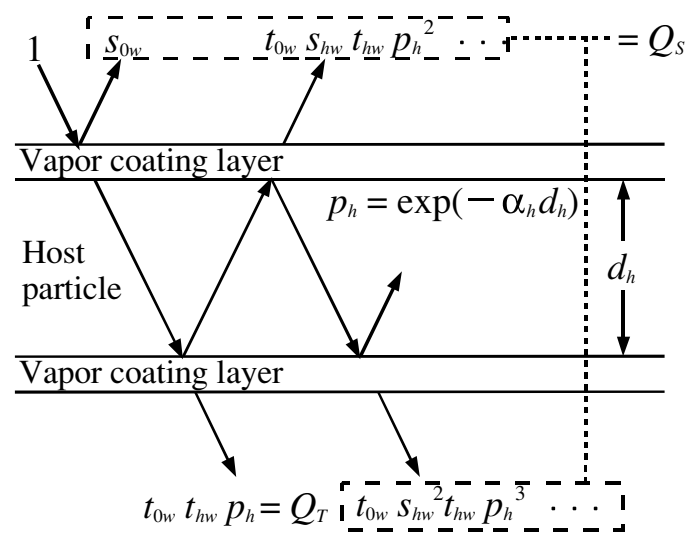

Fig. 3. A model scheme for calculating the scattering and transmitting efficiencies $Q_{S}$ and $Q_{T}$ from internal and external reflectances and transmittances in Fig. 2 , and the absorption coefficient $\alpha_{h}$ and thickness $d_{h}$ of the host material.

In this way, the forward scattering (transmitting) portion $\left(Q_{T}\right)$ automatically changes according to the changes in grain size and the degree of space weathering, independent of the phase function $(P(g), g$ is the phase angle) that is multiplied to the scattering efficiency $\left(Q_{S}\right)$. Because the assumption of isotropic scattering $(P(g)=1)$ applies only to this $Q_{S}$ portion, this model is not really an isotropic one; however, it is an isotropic model which can approximate the real situation of scattering of mineral grains better than the traditional scheme where $Q_{T}$ is not separated from $Q_{S}$. The scattering efficiency $\left(Q_{S}\right)$ and transmitting efficiency $\left(Q_{T}\right)$ are calculated from Fig. 3 as:

$$
\begin{aligned}
Q_{S} & =s_{0 w}+\frac{t_{0 w} s_{h w} t_{h w} p_{h}^{2}}{1-s_{h w} p_{h}} \\
Q_{T} & =t_{0 w} t_{h w} p_{h} \\
p_{h} & =\exp \left(-\alpha_{h} d_{h}\right)
\end{aligned}
$$

The single scattering albedo $w$ is given as:

$$
w=Q_{S} / Q_{E}
$$

Based on Hapke (1993), it is assumed that the bidirectional reflectance $R$ of a particulate medium with no prefer- 
ential escape of singly scattered light is expressed as:

$$
\begin{aligned}
R\left(\theta_{i}, \theta_{e}, \theta_{g}, w\right) & =\frac{w}{4} \frac{1}{\mu_{i}+\mu_{e}} H\left(\mu_{i}, w\right) H\left(\mu_{e}, w\right), \\
\mu_{i} & =\cos \theta_{i}, \quad \mu_{e}=\cos \theta_{e}
\end{aligned}
$$

where $\theta_{i}, \theta_{e}$, and $\theta_{g}$ are incidence, emergence, and phase angles, respectively. The $H$ function is given as:

$$
\begin{aligned}
H(\mu, w)= & {\left[1-(1-\sqrt{1-w}) \mu\left\{r_{0}+\left(1-\frac{1}{2} r_{0}\right.\right.\right.} \\
& \left.\left.\left.-r_{0} \mu\right) \ln \left(1+\frac{1}{\mu}\right)\right\}\right]^{-1}, \\
r_{0}= & \frac{2}{1+\sqrt{1-w}}-1 .
\end{aligned}
$$

Then, the calculated reflectance $R_{C}$ relative to a perfect reflector is:

$$
R_{C}=\frac{R\left(\theta_{i}, \theta_{e}, \theta_{w}\right)}{R\left(\theta_{i}, \theta_{e}, \theta_{g}, w=1\right)} .
$$

Because the measured reflectance (denoted as $R_{M}$ ) may have some other darkening factors, such as surface roughness and other space weathering processes, a coefficient $c$ is multiplied to the $R_{C}$ to fit with $R_{M}$ :

$$
R_{M} \cong c R_{C}
$$

\section{Application to Lunar Soil Suites}

Our new modeling approach has been applied to the bidirectional reflectance spectra of four suites of Apollo lunar soils (Fischer and Pieters, 1994; Fischer, 1995). These soils have similar bulk chemistry and mineralogy but different degrees of space weathering within each suite (Table 1). Their spectra were measured under the same viewing geometry of $30^{\circ}$ incidence, $0^{\circ}$ emergence, $30^{\circ}$ phase angles relative to Halon, and a correction was subsequently made for the absorption features of Halon.

The volume fraction $\phi_{w}$ was assumed to be 0.01 for the Apollo 16a suite, and those for the other suites were assumed to be proportional to the average bulk $\mathrm{FeO}$ value of each suite. The vapor coating layer thickness $d_{w}$ was assumed to be $30 \mathrm{~nm}$ for the freshest Apollo 16a soil (61221), and those for the freshest soils in other suites were assumed to be proportional to the $I_{s} / \mathrm{FeO}$ values in Table 1 . The $\phi_{w}$ and $d_{w}$ values of 61221 are assumed based on the fact that 61221 is relatively fresh, with one restriction that the weathering layer has to be thicker than the diameter of the $\mathrm{npFe}^{0}$ particles, which is around $10 \mathrm{~nm}$. The coefficient $c$ in Eq. (16) for the freshest soil within each suite (12030, 14141,61221 , and 67941) was assumed to be 1.0, and the diameter of host material was assumed to be $10 \mu \mathrm{m}$ for all samples. Given the above assumptions, the absorption coefficient spectrum of each host material $\left(\alpha_{h}\right)$ of the freshest soil in each suite was calculated, based on which $d_{w}$ and $c$ values for the other soils within the same suite were optimized for the best fit with the measured reflectance spectra. The refractive index spectra of host materials $\left(n_{h}(\lambda)\right)$ were assumed to be common and the average for typical olivine and pyroxene (Hiroi and Pieters, 1994):

$$
n_{h}(\lambda)=1.5493+0.066380 / \lambda \text {. }
$$

Table 1. $\mathrm{FeO}\left(\mathrm{wt} \%\right.$ ) and $I_{s} / \mathrm{FeO}$ value of the four lunar soil suites (Fischer and Pieters, 1994; Fischer, 1995).

\begin{tabular}{ccc}
\hline & $\mathrm{FeO}$ & $I_{S} / \mathrm{FeO}$ \\
\hline Apollo 12 & & \\
12030 & 15.40 & 14 \\
12024 & 14.60 & 30 \\
12023 & 15.40 & 60 \\
Apollo 14 & & \\
14141 & 10.20 & 6 \\
14149 & 9.95 & 53 \\
14148 & 10.55 & 74 \\
Apollo $16 \mathrm{a}$ & & \\
61221 & 4.55 & 10 \\
67701 & 4.17 & 37 \\
60051 & 4.50 & 57 \\
64801 & 5.01 & 78 \\
Apollo 16b & & \\
67941 & 4.73 & 29 \\
61241 & 5.33 & 47 \\
68501 & 5.48 & 85 \\
\hline
\end{tabular}

Wavelength-dependent refractive indices of Fe were taken Johnson and Christy (1974), and linear interpolation and extrapolation were performed to cover the necessary wavelength range.

The spectral fitting results are plotted in Fig. 4, the $d_{w}$ values are plotted in Fig. 5 against $I_{s} / \mathrm{FeO}$, which supposedly indicates the degree of space weathering (Morris, 1977), and the absorption coefficient spectra of host materials are plotted in Fig. 6. These results show that reflectance spectra of lunar soils of different maturity can be reasonably reproduced if given a suite of compositionally similar soils and if the derived coating thickness has a good positive correlation with $I_{s} / \mathrm{FeO}$, with the exception of the Apollo 14 suite (Fig. 5). However, actual grains have variable thicknesses of coatings, with some grains having no coating and others having thicker or thinner coating; therefore, the $d_{w}$ values in Fig. 5 show representative values. Poorer spectral fits for the Apollo 12 and 14 suites than for the Apollo 16 suites in Fig. 4 as well as the fact that the $c$ value is significantly smaller than 1.0 may indicate that there are products in addition to vapor coatings that result from space weathering processes and affect the spectra. In lunar soils, the most voluminous space weathering product tends to be agglutinates, which may make up as much as $60 \mathrm{vol} \%$ of a mature lunar soil (McKay et al., 1991). The effect of agglutinates on the reflectance spectra of lunar soils is supposedly included in the $c$ parameter in Eq. (16), which has a correlation with the degree of space weathering. However, its effect needs to be investigated further in future studies.

The absorption coefficient spectra in Fig. 6 show a good correlation between the bulk Fe content and the strength of the absorption band. However, the increasing background continuum along with the increasing bulk Fe content may indicate an incomplete removal of the surface scattering effect due to the assumption that the refractive index $n$ is common for all soil materials, regardless of their Fe contents. Still, these absorption spectra are possibly ideal for applying a deconvolution technique, such as the modified Gaus- 

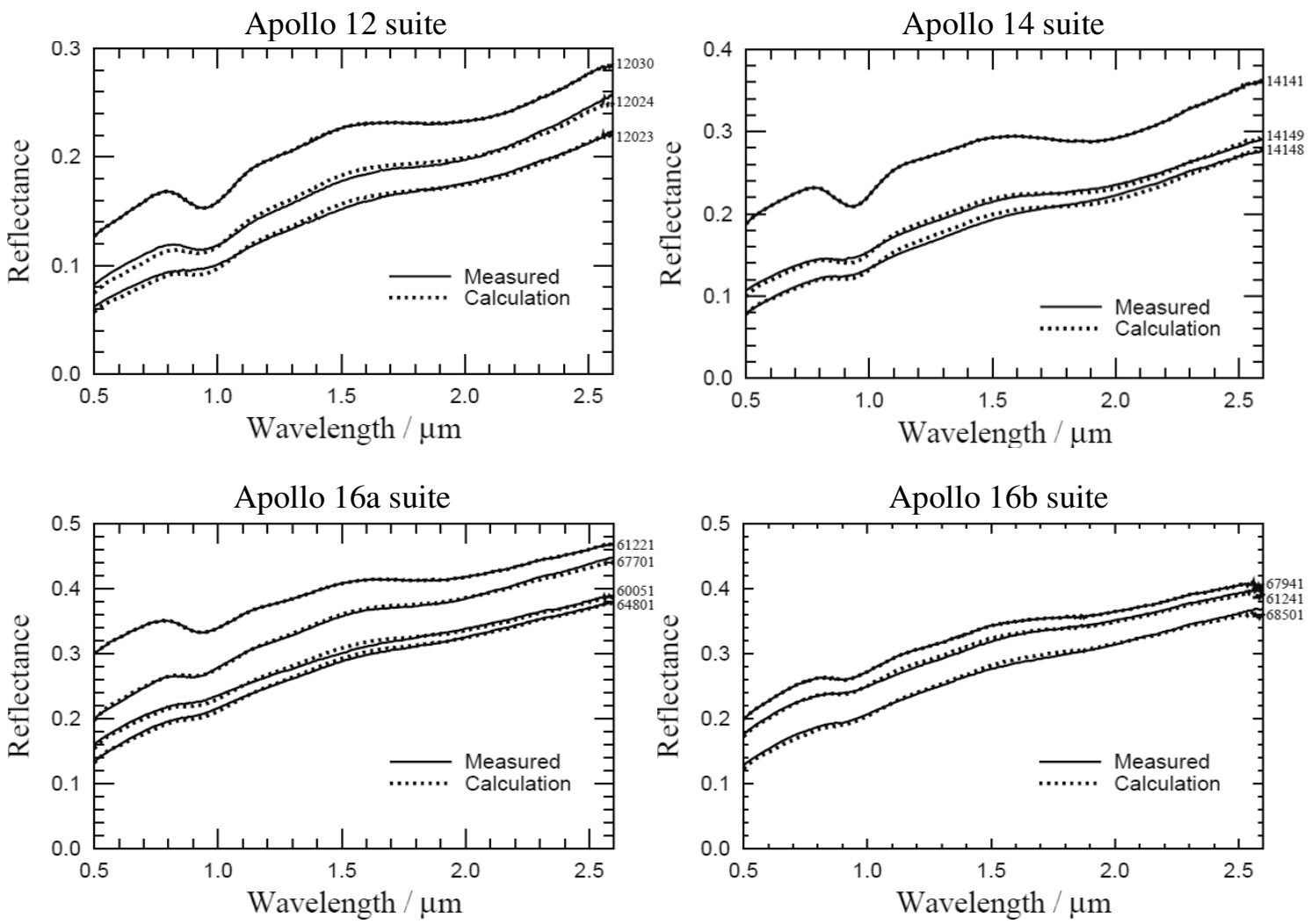

Fig. 4. Model fitting of four Apollo soil suites. Each plot shows the spectra of a soil suite having similar compositions but different maturity (Fischer and Pieters, 1994; Fischer, 1995). The solid and broken lines indicate measured and model spectra respectively.

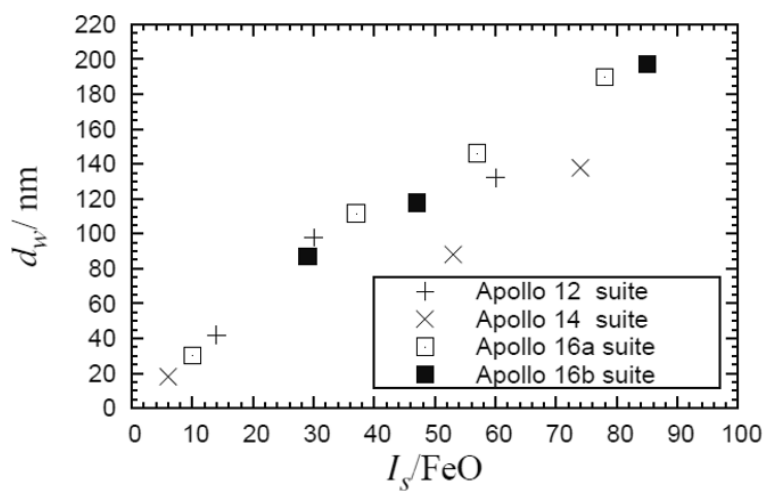

Fig. 5. Optimized values of coating thickness plotted against the $I_{S} / \mathrm{FeO}$ for four Apollo soil suites.

sian model (Sunshine et al., 1990). The absorption band strengths around 1 and $2 \mu \mathrm{m}$ appear to have a good correlation with the bulk $\mathrm{FeO}$ contents.

\section{Conclusions}

A new scheme of applying Hapke's space weathering model has been proposed which accounts for the change in the surface reflectivity of a vapor-coated particle. According to the initial application to four suites of Apollo soils, this model can provide reasonable estimates of the total amount of $\mathrm{nFe}^{0}$ particles formed in the coating layer as well as the absorption coefficient spectrum of the host material fit for analyzing its composition. Future improvements are expected to increase its usefulness, especially for future

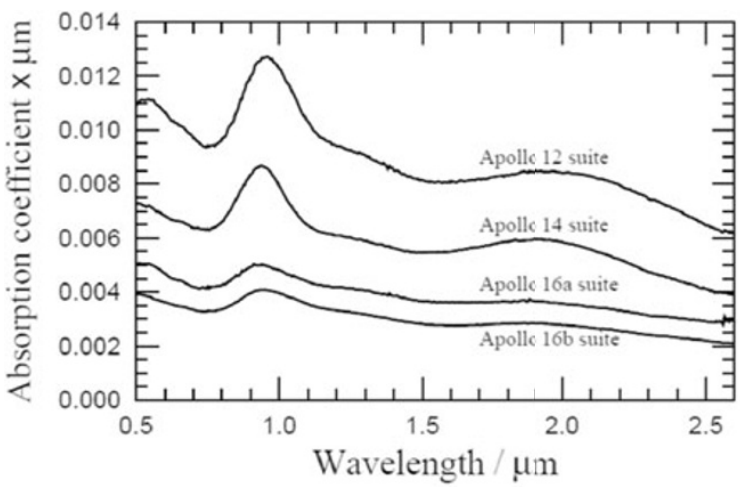

Fig. 6. The absorption coefficient spectra of four lunar soils derived from model calculations.

lunar missions equipped with a spectrometer with a wide wavelength range and a high spectral resolution, such as the Spectral Profiler onboard SELENE/KAGUYA and the $\mathrm{M}^{3}$ onboard Chandrayaan-1.

Acknowledgments. Special thanks to Sarah Noble for many important suggestions on space-weathering of lunar soils. We also thank Drs. Bruce Hapke and Sarah Noble for constructive reviews. T. N. appreciates the financial support by the Mie International Exchange Foundation. T. H. was partially supported by NASA Discovery Data Analysis Program.

\section{References}

Fischer, E. M., Quantitative compositional analysis of the lunar surface from reflectance spectroscopy: Iron, aluminum, and a model for remov- 
ing the optical effects of space weathering, Ph.D. Thesis, 1995.

Fischer, E. M. and C. M. Pieters, Remote determination of exposure degree and iron concentration of lunar soils using Vis-NIR spectroscopic methods, Icarus, 111, 475-488, 1994.

Hapke, B., Bidirectional reflectance spectroscopy 1: Theory, J. Geophys. Res., 86, 3039-3054, 1981.

Hapke, B., Theory of Reflectance and Emittance Spectroscopy, Cambridge Univ. Press, New York, 1993.

Hapke, B., Space weathering from Mercury to the asteroid belt, J. Geophys. Res., 106, 10039-10073, 2001.

Hiroi, T. and C. M. Pieters, Estimation of grain sizes and mixing ratios of fine powder mixtures of common geologic minerals, J. Geophys. Res., 99, 10867-10879, 1994.

Johnson, P. B. and R. W. Christy, Optical constants of transition metals: Ti, V, Cr, Mn, Fe, Co, Ni, and Pd, Phys. Rev. B, 9, 5056-5070, 1974.

Keller, L. P. and D. S. McKay, Discovery of vapor deposits in the lunar regolith, Science, 261, 1305-1307, 1993.

Keller, L. P. and D. S. McKay, The nature and origin of rims on lunar soil grains, Geochim. Cosmochim. Acta, 61, 2331-2341, 1997.

McKay, D. S., G. H. Heiken, A. Basu, G. Blanford, S. Simon, R. Reedy, B. M. French, and J. Papike, The lunar regolith, in The lunar sourcebook, edited by G. H. Heiken, D. T. Vaniman, and B. M. French, pp. 285-356, Cambridge University Press, New York, 1991.
Morris, R. V., Origin and evolution of the grain-size dependence of the concentration of fine-grained metal in lunar soils: The maturation of lunar soils to a steady-state stage, Lunar Planet Sci, 8, 3719-3747, 1977.

Noble, S. K., C. M. Pieters, and L. P. Keller, Can space weathering survive lithification? Results of a TEM study of lunar regolith breccia 10068, Lunar Planet. Sci., 33, no. 1334, 2002.

Pieters, C. M., E. M. Fischer, O. Rode, and A. Basu, Optical effects of space weathering: The role of the finest fraction, J. Geophys. Res., 98, 20817-20824, 1993.

Sasaki, S., K. Nakamura, Y. Hamabe, E. Kurahashi, and T. Hiroi, Production of iron nanoparticles by laser irradiation in a simulation of lunarlike space weathering, Nature, 410, 555-557, 2001.

Sunshine, J. M., C. M. Pieters, and S. F. Pratt, Deconvolution of mineral absorption bands: An improved approach, J. Geophys. Res., 95, 69556966, 1990.

Wentworth, S. J., L. P. Keller, D. S. McKay, and R. V. Morris, Space weathering on the moon: Patina on Apollo 17 samples 75075 and 76015 , Meteorit. Planet. Sci., 34, 593-603, 1999.

T. Nimura (e-mail: nimura@planeta.sci.isas.jaxa.jp), T. Hiroi, and C. M. Pieters 